\title{
Indications for laboratory tests in primary care: assessment of the most frequent indications and requests with blank clinical information
}

\author{
Maria Salinas ${ }^{* 1,2}$, Maite López-Garrigós ${ }^{1}$, Emilio Flores ${ }^{1,3}$, Maria Leiva-Salinas², Patricia Esteban1, Miguel Ahumada3 ${ }^{3}$, Carlos Leiva-Salinas ${ }^{4}$ \\ ${ }^{1}$ Clinical Laboratory, Hospital Universitario de San Juan, San Juan de Alicante, Spain \\ ${ }^{2}$ Department of Biochemistry and Molecular Pathology, Universidad Miguel Hernandez, Elche, Spain \\ ${ }^{3}$ Department of Clinical Medicine, Universidad Miguel Hernandez, Elche, Spain \\ ${ }^{4}$ Department of Radiology, University of Virginia, Charlottesville, USA \\ *Corresponding author: salinas_mar@gva.es
}

\begin{abstract}
Introduction: The aim of this work is twofold. Firstly, to study the temporal evolution in the number of laboratory requests from primary care without clinical indication, and to analyse the number of such requests before and after the implementation of an automated requesting procedure. Secondly, to investigate what are the most frequent clinical indications that prompted laboratory testing.

Materials and methods: This is a retrospective observational study conducted from January 2009 to December 2015. We counted the requests without clinical question, calculated the number of such requests per total number of requests and listed the most frequent indications.

Results: The number of tests requests with a blank clinical indication was significantly higher in 2009 when compared to 2015 (80\% vs. $20 \%$; $P<$ 0.001). For every year in this 7-year period, dyslipidemia, essential hypertension and diabetes were the most prevalent diagnoses that prompted a laboratory test in primary care, accounting for more than $20 \%$ of all indications.

Conclusions: The number of primary care requests without patient clinical question has decreased after the implementation of an automated requesting procedure. Disorders of lipid metabolism, essential hypertension and diabetes mellitus were the most prevalent diagnoses that prompted a laboratory test in primary care.
\end{abstract}

Key words: clinical laboratory; pre-analytical phase; primary care; quality indicators; test request

\section{Introduction}

Failure to order appropriate diagnostic tests, including laboratory tests, accounts for approximately $55 \%$ of observed incidents of missed and delayed diagnoses in the outpatient setting $(1,2)$. Since the laboratory professional should be involved in every step of total testing process, it is important for us to be focused on these events for a possible solution.

One of the main duties of health care workers is to ensure appropriate use of diagnosis and treatment procedures, and to orient health care organizations to more efficient and safety procedures. In fact, there are many advantages regarding an ap- propriate requesting. First, it reduces the economic costs of the laboratory. Second, the incidence of false positive results by the fact of being requested in a population with low prevalence of a certain disease could be significantly reduced (3). Third, it decreases the global request of laboratory tests; indeed inappropriate over requesting may have contributed to the considerably increase in the volume of laboratory tests over the last years. This overload can mask the really clinical important laboratory information and causes the laboratory to be commoditized in a way that it is very difficult to pay the necessary attention to really appropri- 
ate requested tests, to deliver meaningful clinical laboratory information instead of simply laboratory numerical data (4).

In all, knowing the clinical indication for ordered tests is crucial in assessing appropriateness of the ordered test. The Model of Quality Indicators (MQI) proposed by the IFCC Working Group "Laboratory Errors and Patient Safety" (IFCC WG-LEPS) for the pre-analytical phase, as a quality assurance tool for improving the quality of laboratory service, includes quality indicators (QIs) regarding appropriateness of clinical request related to the number of requests without clinical question (5). The priority scale for proposed Qls to facilitate their gradual introduction into routine practice, starts with a "mandatory" (score 1) and ends with a "valuable" (score 4) Qls score. The number of requests without clinical question QI is classified as important (score 2), and should be implemented when all "mandatory" QIs are in use (5). To the best of our knowledge no prior research has dealt with this topic.

The aim of this work is twofold. Firstly, we aimed to study the temporal evolution in the number of laboratory requests from primary care without clinical indication, and to analyse the number of such requests before and after the implementation of an automated requesting procedure. Secondly, we wanted to investigate what are the most frequent clinical indications that prompt a laboratory test.

\section{Materials and methods}

\section{Study design}

A retrospective observational study was conducted from January 2009 to December 2015 in the Health Department (HD) of San Juan (Alicante, Spain). The laboratory is located at the public University Hospital of San Juan, a 370-bed suburban community hospital that serves the HD population (234,551 inhabitants), including nine different primary care centres (PCC). It receives samples from inpatients, outpatients and primary care patients. Primary care samples are transported by couriers from the different PCC to the laboratory reception desk.
Prior to May 2009, primary care tests ordering request was manual (paper request form), and patient demographic data and tests were registered manually in the laboratory information system (LIS) by two laboratory administrative assistants, after receiving the forms in the laboratory reception desk together with samples. Since that time, laboratory requests are made through an electronic system, the Computerized Patient Order Entry (CPOE) that offers the General Practitioners (GPs) a field to be filled regarding the reason for the laboratory request through International Classification of Diseases, Ninth Revision, Clinical Modification (CIE-9-MC) codes (6). It was not mandatory to complete the clinical details field in CPOE.

\section{Methods}

We calculated the number of requests without clinical indication and counted the different clinical information/clinical questions based on CIE-9MC codes, which were provided in the request form. We also assessed the number of primary care requests. Data was collected from the LIS using a software program based on Data Warehouse and On-Line Analytical Processing (OLAP) cube (Omnium by Roche Diagnostics_ SUNSET Technologies, Gerona, Spain).

\section{Data processing}

After collecting the data, we calculated the number of requests without clinical question per total number of requests per year and the difference between 2009 and 2015 (5). We also counted the number of requests with every clinical question per year. We included the clinical indications with prevalence above $1 \%$ of the total clinical indications for the year 2015; this threshold was chosen arbitrarily by the authors. The rest were grouped into a category called other diagnosis.

\section{Statistical analysis}

The difference in indicator results of categorical responses between two independent groups was calculated by way of the comparison of proportions and Chi-square. $A$ two-sided $P \leq 0.001$ rule was utilized as the criterion for rejecting the null 
hypothesis of no difference. All analyses were performed using SPSS Inc. for Windows, Version 22.0. (Chicago, SPSS Inc).

\section{Results}

The number of laboratory requests with pertinent clinical information/indication increased over time, especially after the implementation of the CPOE, surpassing $80 \%$ of requests in 2014 and 2015. Figure 1 shows the number of requests without clinical question per total number of requests over the 7 years of the study. The QI - number of requests without clinical question per total number of requests was significantly $(P<0.001)$ higher in 2009 as compared to 2015.

Table 1 shows the total annual number of requests from primary care and those with patient clinical information. For all 7 years of the study period, disorders of lipid metabolism, essential hypertension and diabetes mellitus were the most frequent indications behind a laboratory test (Table 1).

\section{Discussion}

This is the first research that studies the number of laboratory requests from primary care without clinical question and lists the most frequent indications that prompt a laboratory test in this setting. The study highlights an improvement in the number of requests with patient clinical information after establishment of CPOE. This improvement was significant, with a progressive increment over the study period. Consistently through the 7 years of the study, disorders of lipid metabolism, essential hypertension and diabetes mellitus were the most prevalent in primary care.

Our results prove how technology can improve the correct completeness of a laboratory request. It would be difficult to know how this improvement consequently enhanced the service provided by the laboratory, and hence patient benefit. However, having the patient clinical information when verifying laboratory data makes it easier to report numerical data with added interpretation instead of just numerical data in the $70 \%$ of clinical decisions that laboratory intervenes (4).
Percentage of "Number of request without clinical question/ Total number of request"

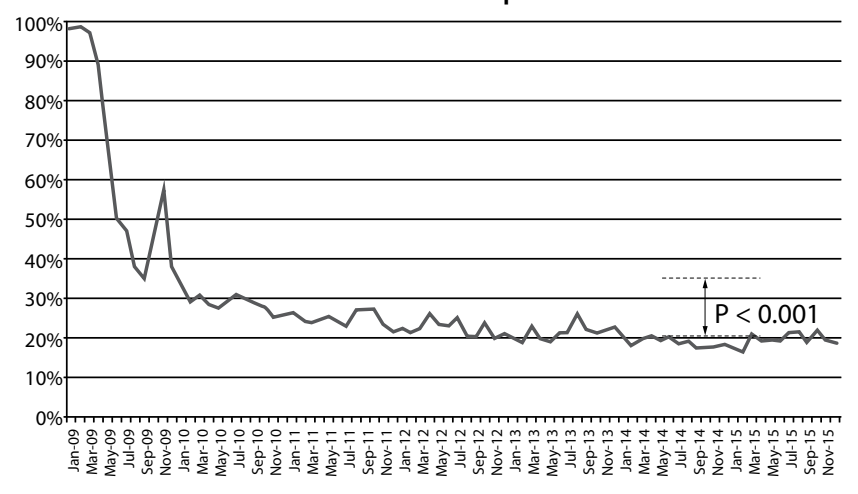

Figure 1. Monthly trend along the 7 years of the indicator number of requests without clinical question per total number of requests

This QI result, after the great improvement in year 2010, also continued improving along years. In fact, in the year 2015 less than 20\% of requests from primary care did not include the pertinent clinical information. There are probably multiple causes for this improvement and it is possible that communication and education (7), as in other stages of laboratory total testing process (8-10), could have contributed to the improvement of GPs awareness for a better request completion.

Patient's clinical information give us the opportunity to enhance patient benefit through laboratory post analytical step improvement by reporting numerical data with added interpretation instead of just numerical data, but also to improve adequacy in test requesting. Consistently, through the 7 years of the study disorders of lipid metabolism, essential hypertension and diabetes mellitus were the most frequent indications behind a laboratory tests. In 2014 and 2015, more than 20\% of tests were performed for one of those reasons. Through interventions, the laboratory could offer the best services to those $20 \%$ of primary care patients, just by acting in such three main patient diagnoses. The added value of the research is that the knowledge of the indications behind laboratory tests allows pathologists and GPs to design and implement tests profiles on the basis of the most common clinical concerns, to make sure all but only the appropriate tests are performed for a certain condition. 
TABLE 1. Total requests, requests with clinical question from primary care and percentage of every diagnosis every year.

\begin{tabular}{|c|c|c|c|c|c|c|c|}
\hline \multirow{2}{*}{ Indicator } & \multicolumn{7}{|c|}{ Year } \\
\hline & 2009 & 2010 & 2011 & 2012 & 2013 & 2014 & 2015 \\
\hline Total requests, $\mathbf{N}$ & 89,450 & 87,056 & 91,676 & 87,424 & 94,922 & 97,438 & 95,017 \\
\hline $\begin{array}{l}\text { Requests with clinical } \\
\text { question, } \mathbf{N}(\%)^{*}\end{array}$ & $\begin{array}{l}31,296 \\
(35.0 \%)\end{array}$ & $\begin{array}{l}62,028 \\
(71.3 \%)\end{array}$ & $\begin{array}{l}69,018 \\
(75.3 \%)\end{array}$ & $\begin{array}{l}67,556 \\
(77.3 \%)\end{array}$ & $\begin{array}{l}74,701 \\
(78.7 \%)\end{array}$ & $\begin{array}{l}79,057 \\
(81.1 \%)\end{array}$ & $\begin{array}{c}76,372 \\
(80.4 \%)\end{array}$ \\
\hline \multicolumn{8}{|l|}{ CIE - code ${ }^{\dagger}$} \\
\hline 272 & $3.9 \%$ & $7.7 \%$ & $7.7 \%$ & $7.9 \%$ & $9.6 \%$ & $9.8 \%$ & $9.1 \%$ \\
\hline 401 & $2.7 \%$ & $5.2 \%$ & $5.2 \%$ & $5.6 \%$ & $6,2 \%$ & $6.0 \%$ & $5.7 \%$ \\
\hline 250 & $1.8 \%$ & $3.7 \%$ & $3.8 \%$ & $4.1 \%$ & $5.1 \%$ & $5.4 \%$ & $5.6 \%$ \\
\hline 595 & $1.0 \%$ & $2.2 \%$ & $2.3 \%$ & $2.3 \%$ & $2.7 \%$ & $3.4 \%$ & $3.2 \%$ \\
\hline 780 & $2.0 \%$ & $3.8 \%$ & $2.6 \%$ & $2.5 \%$ & $2.5 \%$ & $2.7 \%$ & $2.8 \%$ \\
\hline 244 & $0.7 \%$ & $1.5 \%$ & $1.6 \%$ & $1.9 \%$ & $2.3 \%$ & $2.5 \%$ & $2.7 \%$ \\
\hline 280 & $0.6 \%$ & $1.1 \%$ & $1.1 \%$ & $1.3 \%$ & $1.4 \%$ & $1.5 \%$ & $1.7 \%$ \\
\hline 285 & $0.5 \%$ & $1.0 \%$ & $1.2 \%$ & $1.3 \%$ & $1.5 \%$ & $1.6 \%$ & $1.7 \%$ \\
\hline 300 & $1.1 \%$ & $2.0 \%$ & $1.9 \%$ & $1.9 \%$ & $1.6 \%$ & $1.6 \%$ & $1.6 \%$ \\
\hline 790 & $0.5 \%$ & $1.0 \%$ & $1.1 \%$ & $1.2 \%$ & $1.3 \%$ & $1.5 \%$ & $1.5 \%$ \\
\hline 599 & $0.3 \%$ & $0.6 \%$ & $0.8 \%$ & $1.3 \%$ & $1.4 \%$ & $1.6 \%$ & $1.5 \%$ \\
\hline V72 & $0.3 \%$ & $0.6 \%$ & $0.8 \%$ & $1.1 \%$ & $1.1 \%$ & $1.3 \%$ & $1.3 \%$ \\
\hline 536 & $0.4 \%$ & $0.9 \%$ & $1.1 \%$ & $1.5 \%$ & $1.2 \%$ & $1.4 \%$ & $1.3 \%$ \\
\hline 789 & $0.4 \%$ & $1.1 \%$ & $1.2 \%$ & $1.3 \%$ & $1.2 \%$ & $1.3 \%$ & $1.2 \%$ \\
\hline 719 & $0.5 \%$ & $1.2 \%$ & $1.2 \%$ & $1.2 \%$ & $1.2 \%$ & $1.1 \%$ & $1.2 \%$ \\
\hline 788 & $0.4 \%$ & $0.8 \%$ & $0.9 \%$ & $0.9 \%$ & $1.1 \%$ & $1.1 \%$ & $1.1 \%$ \\
\hline 724 & $0.7 \%$ & $1.3 \%$ & $1.4 \%$ & $1.4 \%$ & $1.2 \%$ & $1.1 \%$ & $1.0 \%$ \\
\hline Rest of codes & $17.1 \%$ & $35.6 \%$ & $39.1 \%$ & $38.6 \%$ & $36.1 \%$ & $36.2 \%$ & $36.0 \%$ \\
\hline
\end{tabular}

*The indicator was significantly $(P<0.001)$ lower in year 2009 when compared to $2015 . P<0.05$ was considered statistically significant.

†Full code title: $\mathbf{2 7 2}$ - disorders of lipid metabolism; $\mathbf{4 0 1}$ - essential hypertension; $\mathbf{2 5 0}$ - diabetes mellitus; 595 - cystitis; 780 general symptoms; $\mathbf{2 4 4}$ - acquired hypothyroidism; $\mathbf{2 8 0}$ - iron deficiency anaemia; $\mathbf{7 9 0}$ - nonspecific findings on examination of blood (abnormality of red blood cells, elevated sedimentation rate, abnormal glucose, excessive blood level of alcohol, nonspecific elevation of levels of transaminase or lactic acid dehydrogenase, other abnormal blood chemistry, bacteraemia, viremia, other nonspecific findings on examination of blood); $\mathbf{3 0 0}$ - anxiety, dissociative and somatoform disorders; $\mathbf{2 8 5}$ - other and unspecified anaemias; V72 - special investigations and examinations (examination of eyes and vision, examination of ears and hearing, dental examination, gynaecological examination, pregnancy examination or test, radiological examination, not elsewhere classified, laboratory examination, diagnostic skin and sensitization tests, other specified examinations, unspecified examination); $\mathbf{5 9 9}$ other disorders of urethra and urinary tract; $\mathbf{5 3 6}$ - disorders of function of stomach; $\mathbf{7 8 9}$ - other symptoms involving abdomen and pelvis; 719 - other and unspecified disorders of joint; 724 - other and unspecified disorders of back; 788 - symptoms involving urinary system.

The study had certain limitations. First, we really do not know if - prior to CPOE establishment - the GPs were not indicating the clinical question when filling a laboratory request or the laboratory assistants were not registering this information in the LIS. Second, the period before CPOE, we only had about 20\% of known indications, and the most frequent indications would not have been completely objective.

In conclusion, the number of primary care requests without patient clinical question (measured through a QI) has decreased along years and after 
the implementation of a CPOE. Education and communication between laboratory department and GPs has also contributed to this significantly decrease. Disorders of lipid metabolism, essential hypertension and diabetes mellitus were the most

\section{References}

1. Hickner J, Graham DG, Elder NC, Brandt E, Emsermann CB, Dovey $S$, et al. Testing process errors and their harms and consequences reported from family medicine practices: $a$ study of the American Academy of Family Physicians National Research Network. Qual Saf Health Care 2008;17:194200. http://dx.doi.org/10.1136/qshc.2006.021915.

2. Wahls TL, Cram PM. The frequency of missed test results and associated treatment delays in a highly computerized health system. BMC Fam Pract 2007;8:32-42. http://dx.doi. org/10.1186/1471-2296-8-32.

3. Salinas M, Lopez-Garrigós M, Flors L, Leiva-Salinas C. Laboratory false-positive results: a clinician responsibility or a shared responsibility with requesting clinicians? Clin Chem Lab Med 2013;51:e199-200. http://dx.doi.org/10.1515/ cclm-2013-0043.

4. Salinas M, López-Garrigós M, Uris J. Towards laboratory knowledge, not data, in 70\% of clinical decision-making. What "knowledge management" can add to clinical practice? Clin Chem Lab Med 2011;49:1389-90. http://dx.doi. org/10.1515/CCLM.2011.636.

5. Plebani M, Sciacovelli L, Aita A, Chiozza ML. Harmonization of pre-analytical quality indicators. Biochem Med (Zagreb) 2014;24:105-13. http://dx.doi.org/10.11613/BM.2014.012. prevalent diagnoses that prompted a laboratory test in primary care.

\section{Potential conflict of interest}

None declared.

6. Instituto de Información Sanitaria. Boletines de Codificación Clínica con la CIE - 9-MC. Madrid. Ministerio de Sanidad, Servicios Sociales e Igualdad. Available from: http:// www.msssi.gob.es/estadEstudios/estadisticas/normalizacion/clasifEnferm/boletines/home.htm. [In Spanish.] Accessed March 1st 2016.

7. Salinas M, López-Garrigós M, Flores E, Leiva-Salinas M, Ahumada $M$, Leiva-Salinas $C$. Education and communication is the key for the successful management of vitamin $D$ test requesting. Biochem Med (Zagreb) 2015;25:237-41. http:// dx.doi.org/10.11613/BM.2015.024.

8. Salinas $M$, López-Garrigós $M$, Asencio A, Leiva-Salinas $M$, Lugo J, Leiva-Salinas C. Laboratory utilization improvement through a computer-aided algorithm developed with general practitioners. Clin Chem Lab Med 2015;53:1391-7. http:// dx.doi.org/10.1515/cclm-2014-0762.

9. Lillo $R$, Salinas $M$, Lopez-Garrigos $M$, Naranjo-Santana $Y$, Gutiérrez M, Marín MD, et al. Reducing preanalytical laboratory sample errors through educational and technological interventions. Clin Lab 2012;58:911-7.

10. Salinas M, Lopez-Garrigos M, Gutierrez M, Lugo J, Uris J. Two minutes of monthly monitoring can ensure quality laboratory service every day of the year. Lab Medicine 2010;41:1215. $h$ ttp://dx.doi.org/10.1309/LM8OI14LCORJYHVY. 